\title{
Nonlinear optical response of GaN layers on sapphire: The impact of fundamental beam interference
}

\author{
I. V. Kravetsky and I. M. Tiginyanu \\ Institute of Applied Physics, Technical University of Moldova, 2004 Chisinau, Moldova \\ Ralf Hildebrandt ${ }^{a)}$ and Gerd Marowsky \\ Laser-Laboratorium Göttingen e. V., Hans-Adolf-Krebs-Weg 1, D-37077 Göttingen, Germany \\ D. Pavlidis and A. Eisenbach \\ Solid State Electronics Laboratory, The University of Michigan, Ann Arbor, Michigan 48109-2122 \\ H. L. Hartnagel \\ Institut für Hochfrequenztechnik, Technische Universität Darmstadt, D-64283 Darmstadt, Germany
}

(Received 17 August 1999; accepted for publication 16 December 1999)

\begin{abstract}
GaN layers grown by metalorganic chemical-vapor deposition were characterized by optical second- and third-harmonic generation techniques. The angular dependence of the second-harmonic intensity in transmission showed a $c$-textured growth of the GaN layers on the sapphire substrates. The measured ratios $d_{33} / d_{15}$ and $d_{33} / d_{31}$ are equal to -2.02 and -2.03 , respectively, which is indicative of a wurzite structure of the GaN layers. The measured $d_{33}$ is 33 times that of the $d_{11}$ of quartz. Fine oscillations were observed in the measured second- and third-harmonic angular dependencies that are explained by taking into account the interference of the fundamental beam in the GaN/sapphire structure. (C) 2000 American Institute of Physics. [S0003-6951(00)03907-3]
\end{abstract}

Gallium nitride $(\mathrm{GaN})$ is a wide-band-gap semiconductor $\left(E_{g}=3.4 \mathrm{eV}\right.$ at $\left.300 \mathrm{~K}\right)$ with potential applications in hightemperature/high-power electronics and optoelectronics. ${ }^{1} \mathrm{Be}$ ing alloyed with $\mathrm{InN}$ and $\mathrm{AlN}, \mathrm{GaN}$ can be tailored for manufacturing optoelectronic devices covering both visible and UV regions of the spectrum. A subject of particular interest, in this regard, is the nonlinear-optical response of $\mathrm{GaN}$ which was found to be more than 50 times higher than that of quartz. ${ }^{2,3}$ Several second-harmonic generation (SHG) and third-harmonic generation (THG) studies of GaN epilayers have been reported recently. ${ }^{4-6}$ However, no attention was paid to the differences between front and back cases for excitation nor to the angular step resolution on the nonlinear optical response. In this letter, we report on the study of nonlinear optical effects in GaN/sapphire samples as a function of excitation geometry and angular step resolution. Both the second-harmonic ( $\mathrm{SH}$ ) and third-harmonic (TH) signals measured in dependence on the incident angle of the fundamental beam were found to be modulated due to the interference of the fundamental beam in the sample.

The GaN layers used in our experiments were grown by low-pressure metalorganic chemical-vapor deposition (MOCVD) on (0001) $c$-plane sapphire using trimethylgallium $(\mathrm{TMGa})$ and ammonia $\left(\mathrm{NH}_{3}\right)$ as source materials. A modified EMCORE GS-3200 system was used for this purpose. A buffer layer of about 25-nm-thick $\mathrm{GaN}$ was first grown at $510^{\circ} \mathrm{C}$. The GaN layers grown on top at temperatures ranging from 850 to $1050{ }^{\circ} \mathrm{C}$ had thicknesses of about 1 $\mu \mathrm{m}$.

Polarized SHG and THG measurements were carried out in transmission mode for both front (the GaN layer facing

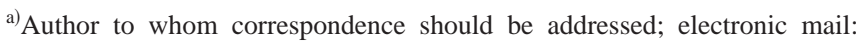
rhildebr@1lg.gwdg.de
}

the incident pump beam) and back (the sapphire substrate facing the incident pump beam) cases. As a fundamental beam, the $1064 \mathrm{~nm}$ output of a $Q$-switched Nd-YAG laser (Spectra Physics GCR-170) with a $10 \mathrm{~Hz}$ repetition rate and $7 \mathrm{~ns}$ pulse width was used. To minimize the influence of the laser output fluctuations, the measured $\mathrm{SH}\left(\lambda_{2 \omega}=532 \mathrm{~nm}\right)$ and TH $\left(\lambda_{3 \omega}=355 \mathrm{~nm}\right)$ intensities were normalized by the simultaneously monitored laser intensity in the reference channel. The sample was mounted on a step-motorized rotation stage. The direction of the fundamental beam polarization was changed by rotating the half-wave plate placed in front of the sample. The fundamental wavelength was filtered out from $\mathrm{SH}$ and $\mathrm{TH}$ signals by using appropriate color filters and a grating monochromator.

The wurzite structure of GaN belongs to the $6 \mathrm{~mm}$ point group symmetry. In this case, there are three nonzero nonlinear-optical coefficients $d_{15}, d_{31}$, and $d_{33}$, which are responsible for the second-order nonlinear optical properties. The induced nonlinear polarization in the GaN film has the following components: ${ }^{7}$

$$
\begin{aligned}
& P_{x}(2 \omega)=2 d_{15} E_{z}(\omega) E_{x}(\omega), \\
& P_{y}(2 \omega)=2 d_{15} E_{z}(\omega) E_{y}(\omega), \\
& P_{z}(2 \omega)=d_{31}\left(E_{x}(\omega)^{2}+E_{y}(\omega)^{2}\right)+d_{33} E_{z}(\omega)^{2} .
\end{aligned}
$$

According to Eqs. (1), SHG is forbidden from a $c$-textured film (having the optical axis $c$ perpendicular to the surface) when the pump beam is incident normal to the film. One can notice that for an ideal wurzite structure the nonzero elements of the second-order susceptibility tensor are related to each other as $d_{15} \approx d_{31}$ and $d_{33} / d_{31} \approx-2.8,9$ 


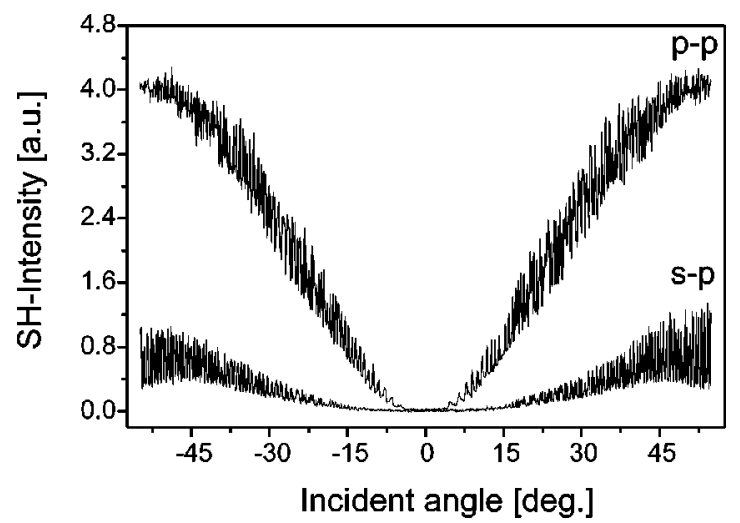

FIG. 1. Measured $p$-polarized second-harmonic intensity in transmission (back case) as a function of the incident angle of the $p$ - and $s$-polarized fundamental beams. The angular resolution is $0.1^{\circ}$ per step.

Neglecting absorption and birefringence of $\mathrm{GaN}$ $\left(\Delta n_{\omega, 2 \omega}=0.02\right),{ }^{2}$ the transmitted SH intensity $I_{2 \omega}$ as a function of the incident angle $\theta$ of the fundamental beam can be written as follows: ${ }^{10,11}$

$$
I_{2 \omega}^{m-p}(\theta) \propto C I_{\omega}^{2}\left(\frac{2 \pi L}{\lambda}\right)^{2}\left(d_{\mathrm{eff}}^{m-p}\right)^{2} \frac{\sin ^{2} \Psi}{\Psi^{2}}
$$

where $I_{2 \omega}^{m-p}$ is the $p$-polarized SH intensity induced by the $m$-polarized fundamental beam (e.g., $s$ or $p$ polarized); $C$ is a parameter determined by the appropriate Fresnel transmission coefficients and the beam area; $L$ is the layer thickness; $\Psi=(2 \pi L / \lambda)\left(n_{\omega} \cos \theta_{\omega}-n_{2 \omega} \cos \theta_{2 \omega}\right)$, where $n_{\omega}$ and $n_{2 \omega}$ are the refractive indices at the fundamental and $\mathrm{SH}$ frequencies in the layer, $\theta_{\omega}$ and $\theta_{2 \omega}$ are the refractive angles of fundamental and SH waves determined by $\sin \theta=n_{\omega} \sin \theta_{\omega}$ and $\sin \theta=n_{2 \omega} \sin \theta_{2 \omega}$, respectively; $d_{\mathrm{eff}}^{m-p}$ are the effective second-order nonlinear-optical coefficients for appropriate polarization combinations. For the transmitted SH intensity the effective second-order nonlinear-optical coefficients are

$$
\begin{aligned}
d_{\mathrm{eff}}^{s-p}= & d_{31} \sin \theta_{2 \omega}, \\
d_{\mathrm{eff}}^{45^{\circ}-p}= & d_{15} \sin \theta_{\omega}, \\
d_{\mathrm{eff}}^{s-p}= & d_{15} \cos \theta_{2 \omega} 2 \theta_{\omega}+d_{31} \cos ^{2} \theta_{\omega} \sin \theta_{2 \omega} \\
& +d_{33} \sin ^{2} \theta_{\omega} \sin \theta_{2 \omega} .
\end{aligned}
$$

The coefficients of $d_{15}, d_{31}$, and $d_{33}$ can be determined by measuring the $\mathrm{SH}$ intensity as a function of the incident angle of the fundamental beam for the above-mentioned polarization combinations and comparing it to the SH intensity of a reference quartz plate.

Figure 1 shows the intensity of the transmitted $p$-polarized SH signal from a $1-\mu \mathrm{m}$-thick $\mathrm{GaN}$ layer on a $326.9 \mu \mathrm{m}$ sapphire substrate as a function of the incident angle of the $p$ - and $s$-polarized fundamental beam for the back case. The measurements were carried out with an angular resolution of $0.1^{\circ}$ per step. In Fig. 1, fine oscillations on the curves are seen. Similar oscillations were also observed in SH angular dependencies measured for the front case. The angular dependence of the $p$-polarized SH intensity measured for the back case with an angular step of $0.01^{\circ}$, as depicted in Fig. 2, shows the modulated pattern of the SH signal in more detail.

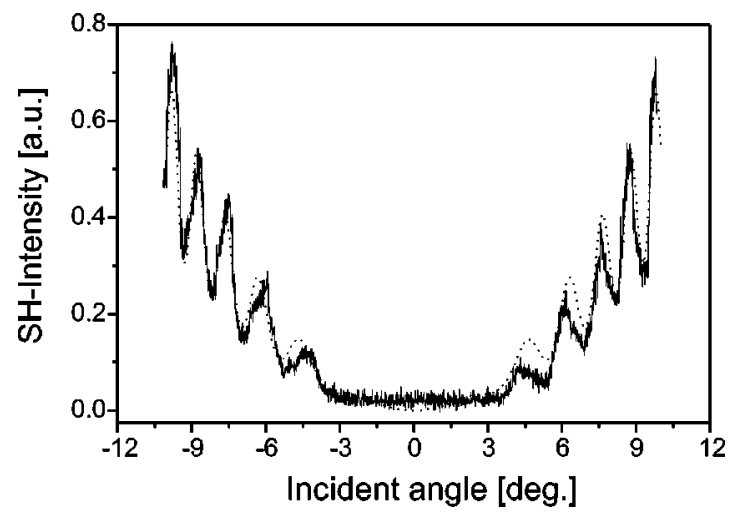

FIG. 2. Measured $p$-polarized second-harmonic intensity in transmission (back case) as a function of the incident angle of the $p$-polarized fundamental beam. The angular resolution is $0.01^{\circ}$ per step. The solid line is experimental, the dotted line is a fit.

The obtained results show that the fine oscillations can be resolved only with an angular step of $0.1^{\circ}$ or smaller. On the other hand, only the observation of the fine oscillations made it possible to reveal the phase shift of $\pi$ between the measured angular dependencies for the front and the back cases. Assuming that the sample possesses two strictly parallel faces (i.e., the sample acts as an interferometer) and taking into account only the interference of the first two transmitted fundamental beams, the pump intensity $I_{\omega}$ can be written as $I_{1}(\omega)+I_{2}(\omega)+2 \sqrt{I_{1}(\omega) I_{2}(\omega)} \cos \delta$, where $I_{1}(\omega)$ is the intensity of the incoming fundamental beam, $I_{2}$ is the intensity of the second transmitted fundamental beam after two reflections inside the sample, and $\delta$ is the phase shift. ${ }^{12}$ After substituting it into Eq. (2), we curve fit the data in Fig. 2. The refractive indices as well as the ratio $I_{2}(\omega) / I_{1}(\omega)$ were fitted parameters. A good fit (dotted line) was obtained with $I_{2}(\omega)=0.007 I_{1}(\omega)$ and the following refractive indices: $n_{\omega}=2.290$ for $\mathrm{GaN}$ and $n_{\omega}=1.755$ for sapphire.

The observed phase shift between the two cases is more clearly seen by measuring the angular dependence of the TH intensity, because in this case THG is allowed at normal incidence. Figure 3 shows the intensity of the transmitted $p$-polarized TH intensity $\left(\lambda_{3 \omega}=355 \mathrm{~nm}\right)$ as a function of the incident angle of the $p$-polarized fundamental beam for both back (solid squares) and front (open circles) cases. Good fits (solid lines) were obtained using the same model of the in-

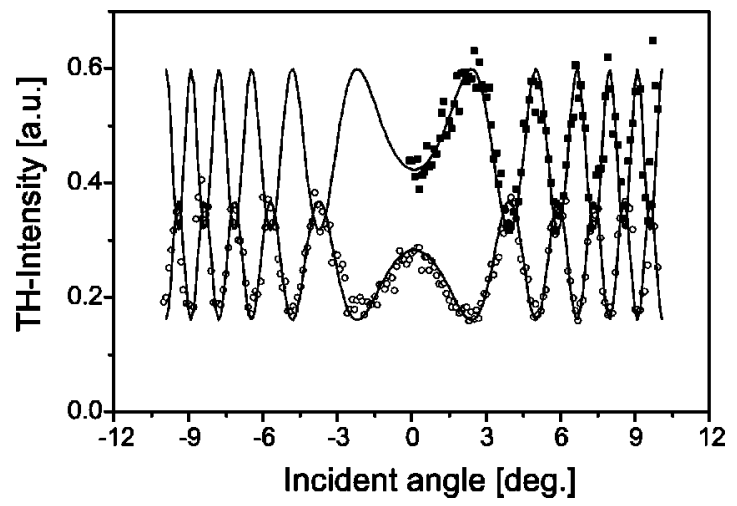

FIG. 3. Measured $p$-polarized third-harmonic intensity in transmission as a function of the incident angle of the $p$-polarized fundamental beam for back (solid squares) and front (open circles) cases, respectively. The angular resolution is $0.1^{\circ}$ per step. Solid lines show fits. 

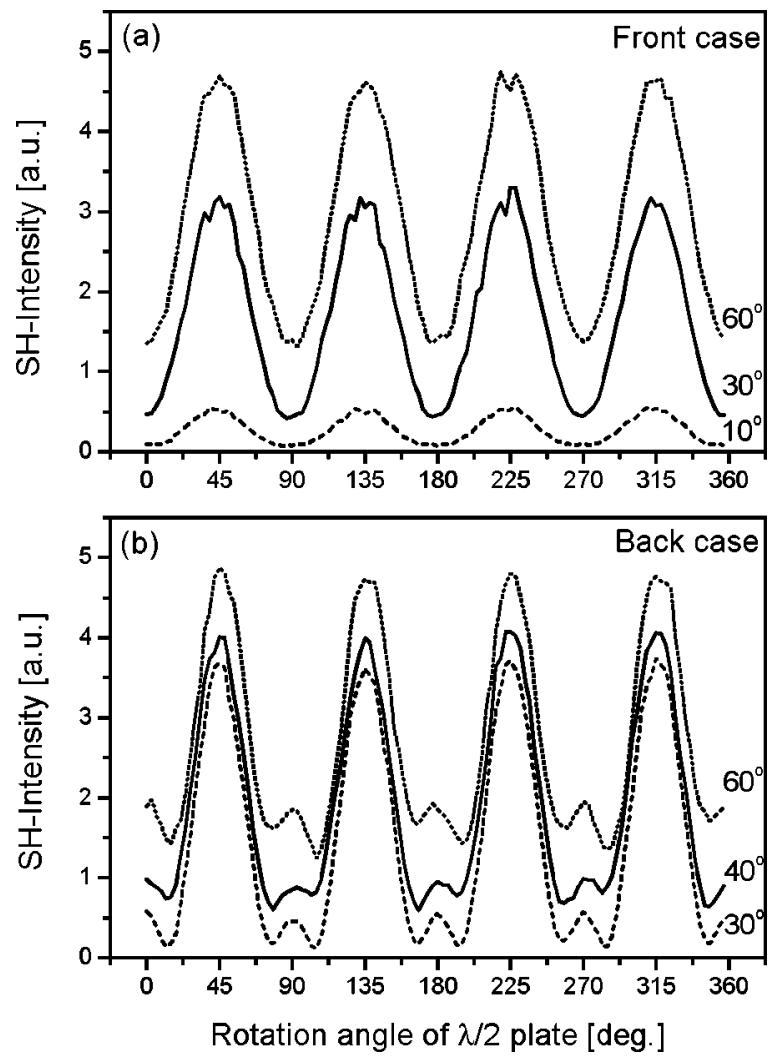

FIG. 4. Measured $p$-polarized second-harmonic intensity in transmission as a function of the rotation angle of the half-wave plate for front (a) and back (b) cases at different incident angles of the fundamental beam $\left(10^{\circ}, 30^{\circ}\right.$, $40^{\circ}$, and $60^{\circ}$ ).

terference of the two fundamental beams in the sample that was applied for the $\mathrm{SH}$ angular dependencies. It should be noted that neither a $\mathrm{SH}$ nor a $\mathrm{TH}$ signal from the sapphire substrate was detected under the experimental conditions.

By comparing Figs. 2 and 3, one can see the equal number of oscillations for both $\mathrm{SH}$ and $\mathrm{TH}$ responses. This and the presented fits suggest that the observed modulation of the nonlinear-optical signal is caused by the interference of the fundamental beam in the sample. In our opinion, the observed oscillations are also indicative of the high quality of the sample.

Comparing the measured $\mathrm{SH}$ intensities for $s-p$ and $45^{\circ}-p$ polarization combinations [see Eqs. (3) and (4)] with the SH Maker fringes of a reference $z$-cut quartz plate $\left[d_{11}(2 \omega)=0.335 \mathrm{pm} / \mathrm{V}\right],{ }^{13}$ the coefficients $d_{15}=5.48 \mathrm{pm} / \mathrm{V}$ and $d_{31}=5.46 \mathrm{pm} / \mathrm{V}$ were deduced. The values of $d_{15}$ and $d_{31}$ relative to $d_{33}$ were found by substituting the obtained values of $d_{15}$ and $d_{31}$ in Eq. (5), and making a least-squares fit of the obtained angular SHG dependence for the $p-p$ polarization combination. We found that $d_{33} / d_{15}=-2.02$ and $d_{33} / d_{31}$ $=-2.03$, and consequently, $d_{33}=-11.07 \mathrm{pm} / \mathrm{V}$. The obtained values are in good agreement with the ones reported in Refs. 4 and 5.

Figure 4 shows the $p$-SH polarization dependencies for the back and front cases at different incident angles of the pump beam. A function of the type $\left(a \cos ^{2} 2 \varphi+b \sin ^{2} 2 \varphi\right)^{2}$ gives a good fit to the curves of the front case (Fig. 4) indicating that $\mathrm{GaN}$ is isotropic in the plane of the film. The $\varphi$ is the rotation angle of the $\lambda / 2$ plate for fundamental radiation ( $\varphi=0^{\circ}$ and $\varphi=45^{\circ}$ correspond to the $s$ - and $p$-polarized fundamental beams, respectively); the parameters $a$ and $b$ are determined by appropriate coefficients $d$ and the transmission factors of the fundamental and $\mathrm{SH}$ radiations. In the back case, the SH signal exhibits additional peaks. This difference is the subject of further studies.

In conclusion, the obtained results confirmed the wurzite structure of the MOCVD GaN layers, with the optical $c$-axis oriented perpendicular to the sapphire substrates. The measured $d_{33}$ is 33 times that of $d_{11}$ of quartz. Using a high angular resolution, we were able to observe fine oscillations in the nonlinear-optical response of GaN. The analysis of the experimental results shows that these oscillations are caused by the interference of the fundamental beam in the sample.

The observed effect of the nonlinear-optical modulation can find potential applications in optoelectronics and might be helpful as a method for the characterization of substrate/ thin-film structures.

Two of the authors (I.V.K. and I.M.T.) gratefully acknowledge the support by the Alexander von HumboldtStiftung. This work was partially supported by the NATO Scientific Division under Grant No. HTECH.LG 961399.

${ }^{1}$ Properties, Processing and Applications of Gallium Nidride and Related Semiconductors, edited by J. H. Edgar, S. Strite, I. Akasaki, H. Amano, and C. Wetzel (1999).

${ }^{2}$ T. Ishidate, K. Inoue, and M. Aoki, Jpn. J. Appl. Phys. 19, 1641 (1980).

${ }^{3}$ H. Y. Zhang, X. H. He, Y. H. Shih, M. Schurman, Z. C. Feng, and R. A. Stall, Appl. Phys. Lett. 69, 2953 (1996).

${ }_{4}^{4}$ J. Miragliotta, D. K. Wickenden, T. J. Kistenmacher, and W. A. Bryden, J. Opt. Soc. Am. B 10, 1447 (1993).

${ }^{5}$ W. E. Angerer, N. Yang, A. G. Yodh, M. A. Khan, and C. J. Sun, Phys. Rev. B 59, 2932 (1999).

${ }^{6}$ J. Miragliotta and D. K. Wickenden, Phys. Rev. B 50, 14960 (1994).

${ }^{7}$ A. Yariv and P. Yeh, Optical Waves in Crystals (Wiley, New York, 1984), pp. 507-510.

${ }^{8}$ B. F. Levine, Phys. Rev. B 7, 2600 (1973).

${ }^{9}$ J. L. Hughes, Y. Wang, and J. E. Sipe, Phys. Rev. B 55, 13630 (1997).

${ }^{10}$ J. Jerphagon and S. K. Kurtz, J. Appl. Phys. 41, 1667 (1970).

${ }^{11}$ W. N. Herman and L. M. Hayden, J. Opt. Soc. Am. B 12, 416 (1995).

${ }^{12}$ E. Hecht, Optics (Addison-Wesley, Reading, MA, 1987), pp. 352-368.

${ }^{13}$ Handbook of Laser Science and Technology, edited by M. J. Weber (CRC, Boca Raton, FL, 1986), Vol. III, Pt. 1, Sec. 1, p. 80. 